\title{
Ramping up RANTES in the acute response to arterial injury
}

\author{
Timothy Hla and Myat Lin Oo
}

Center for Vascular Biology, Department of Pathology and Laboratory Medicine, Weill Medical College of Cornell University, New York, New York, USA.

\begin{abstract}
Arterial injury results in the formation of neointimal lesions. Lack of resolution of the pathologic neointima leads to stenosis, tissue ischemia, and organ dysfunction. In this issue of the JCI, Kovacic et al. show that, in response to arterial injury in mice, the cytokine TNF- $\alpha$ triggers a novel signaling pathway involving the combinatorial action of two transcription factors, STAT3 and NF- $\kappa$ B (p65 subunit), in VSMCs (see the related article beginning on page 303). Upon activation, these factors turn on transcription of a potent $T$ cell chemokine, RANTES, which selectively recruits $T$ cells into the vessel wall as part of the vascular wound-healing response.
\end{abstract}

Blood vessels have myriad functions, including contraction/relaxation, barrier function, thrombosis/hemostasis, and transport. Following injury, complex mechanisms of wound healing and resolution are triggered in order to return the vessel to its optimal functionality $(1,2)$. In fact, it was hypothesized that response to injury is the key factor in determining the evolution of the atherosclerotic plaque into an unstable state that is prone to rupture and subsequent thrombosis (1). This mechanism underlies many instances of acute coronary syndromes, stroke, and peripheral vascular episodes $(3,4)$. Although multiple causes of vascular injury are known (e.g., hyperlipidemia, homocysteinemia, oxidative stress, uncontrolled inflammation, and exposure to tobacco smoke), mechanical injury also causes a repair response in arteries. This type of injury occurs after interventional procedures such as angioplasty and stenting, which are commonly used in the treatment of arterial vessel occlusive diseases. Although stenting is generally initially successful, a significant number of patients experience reocclusion (5), leading to additional invasive procedures and/or bypass surgery. Thus, it is important to understand the cellular and molecular mechanisms underlying remodeling of the arterial vasculature.

\section{Selective recruitment of immune cells by vascular chemokines}

It is established that recruitment of immune cells into the injured vessel is a

Conflict of interest: The authors have declared that no conflict of interest exists.

Citation for this article: J. Clin. Invest. 120:90-92 (2010). doi:10.1172/JCI41738. key event that drives the inflammatory response needed for repair (4). Several types of immune cells, including macrophages and $\mathrm{T}$ cells, are recruited and play catalytic roles in the vascular wound-healing response. Optimal kinetics and the extent of recruitment of various immune cell types are important for proper inflammation and resolution. Thus, the mechanisms involved in these processes have been intensively investigated. As in other tissues, selective expression of chemokines at the site of injury is a principle mechanism that recruits immune cells. For example, it is appreciated that the chemokine CCL2 (also known as monocyte chemoattractant protein 1 [MCP-1]) is expressed by endothelial cells and VSMCs. Antagonism of exaggerated CCL2 function results in attenuated vascular inflammation and restenosis due to reduced macrophage recruitment (6). On the other hand, T cells play important regulatory roles in the inflamed vessel wall. They interact with macrophages and dendritic cells to regulate the inflammatory and wound-healing responses in both positive and negative manners. Thus, some T cell subtypes, such as Th17 cells, are proinflammatory, whereas others, such as Tregs, inhibit inflammatory responses $(7,8)$. Regulation of the influx and egress of $\mathrm{T}$ cells in the injured vascular lesion is not well understood.

\section{A combinatorial mechanism to induce RANTES}

In this issue of the JCI, Kovacic et al. describe a novel mechanism that recruits $T$ cells into injured vessels (9). Using a mouse model of arterial vascular injury, the authors show that $\mathrm{T}$ cell recruitment is driven by VSMC expression of a potent chemokine, RANTES (also known as CCL5). This chemokine signals through the $G$ protein-coupled $T$ cell surface receptor CCR5 to regulate $T$ cell recruitment and amplification of inflammatory reactions $(6,7)$ (Figure 1). Interestingly, VSMC expression of RANTES is driven by the inflammatory cytokine TNF- $\alpha$ and its activation of its receptor TNF- $\alpha$ receptor 1 (TNF- $\alpha-R 1$ ), which subsequently activates the transcription factors NF- $\kappa \mathrm{B}$ (p65 subunit) and STAT3. These findings describe a novel VSMC-specific pathway via which RANTES is induced in the vascular wall in response to injury.

The key protein complex central to this VSMC-specific signaling pathway is com-

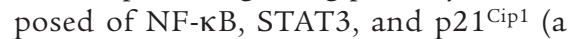
cyclin-dependent kinase inhibitor). Previous studies from the laboratory of Elizabeth Nabel made the key observation that the cell-cycle inhibitor $\mathrm{p} 21^{\mathrm{Cip} 1}$, which is induced in VSMC after vascular injury, inhibits inflammatory gene expression (10). This event effectively coordinates cell-cycle arrest with inflammatory gene expression, thus potentially limiting neointimal hyperplasia. In their current report (9), Kovacic et al. extend this concept further and show the existence of a trimolecular complex composed of NF- $\kappa$ B, STAT3, and $\mathrm{p} 21^{\mathrm{Cip} 1}$ (Figure 1). Indeed, mice that lack $\mathrm{p} 21^{\mathrm{Cip} 1}$ expressed high levels of RANTES and recruited more T cells than the WT mice in response to injury, suggesting that a critical control element is exerted at this juncture in the signaling pathway. In addition, the combinatorial function of two major inflammation-responsive transcriptional activators to induce the RANTES gene is reminiscent of the function of CArG-Box binding factors in the regulation of VSMC differentiation and phenotype switch (11).

\section{Cytokine action on VSMCs regulates chemokines}

Given the importance of the NF-кB/STAT3 pathway, the authors went on to determine the identity of the initiating extracellular 


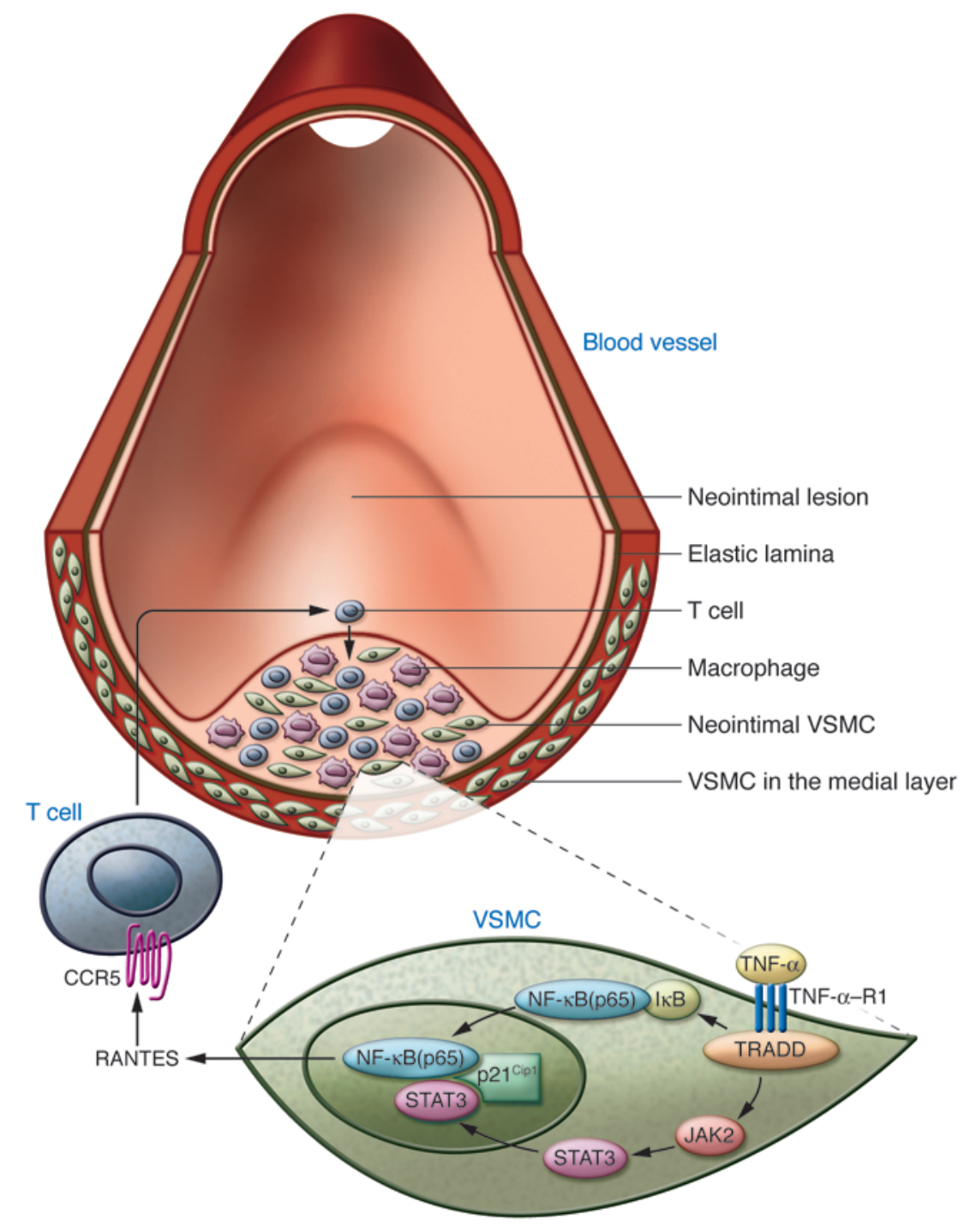

Figure 1

Schematic representation of VSMC signaling mechanisms involved in T cell recruitment following acute arterial injury. During vascular injury and deendothelialization, VSMC migration and activation recruits immune cells such as macrophages and $T$ cells, resulting in the formation of a neointimal lesion. In their study in this issue of the $\mathrm{JCl}$, Kovacic et al. show that TNF- $\alpha$ activation of TNF- $\alpha-R 1$ on VSMCs results in the stimulation of NF- $\kappa B$ (p65 subunit) and STAT3 pathways (9). The complexes formed by NF-KB, STAT3, and p21 cip1 are found in the activated, neointimal VSMCs. Combinatorial action of NF-кB and STAT3 turns on the transcription of the RANTES gene, which encodes the T cell chemokine RANTES (also known as CCL5). Secreted RANTES acts on its receptor, CCR5, on T cells to induce chemotaxis, thus allowing T cell entry into the neointimal lesion. TRADD, TNFRSF1A-associated via death domain.

factor. It turned out that TNF- $\alpha$ is the key cytokine that activates both pathways (Figure 1). While TNF- $\alpha$ activation of NF-кB is well understood (12), VSMC-specific activation of STAT 3 by TNF- $\alpha$ binding to TNF- $\alpha-\mathrm{R} 1$ is surprising and represents economical usage of receptor-dependent signaling pathways in a cell-specific manner. The detailed molecular mechanisms through which injury leads to TNF- $\alpha$ deposition are not clear but may involve monocytes and platelets that are closely associated with vascular elements even under homeostasis $(13,14)$. However, other inflammatory cytokines such as IL- $1 \alpha$ and IL-1 $\beta$ could also be involved, as cytokines induce the expression of each other under inflammatory conditions.
Once RANTES is induced, infiltration of $T$ cells and amplification of inflammatory reactions in the vessel wall would lead to robust neointimal hyperplasia (Figure 1). Optimal recruitment of proper $\mathrm{T}$ cell subtypes may be critical to the repair and reendothelialization processes as well as to the resolution of the lesion $(4,7)$. In the classical immune organs, factors that regulate the influx and egress of $\mathrm{T}$ cell subtypes are better understood (15). It is likely that similar events take place in the inflamed vascular lesions. Further studies are needed to define the interplay of chemokines and $\mathrm{T}$ cell subtypes in neointima formation and involution. However, given the findings of this report (9), it is reasonable to speculate that the VSMC is a key cell type that orchestrates this process.

\section{Conclusions and future perspectives}

A fundamental discovery such as this (9) will undoubtedly stimulate efforts in the search for novel therapeutics to control exaggerated neointimal hyperplasia. Although local delivery of signaling modulators, for example, so-called "drug-eluting stents," has shown promise (16), nonspecificity of the chemical inhibitors and the complex nature of signaling pathways have hampered their rapid deployment. It is important to define cellular and molecular mechanisms in detail not only in animal models but also in clinical and translational studies in humans. For example, it will be important to determine whether the TNF- $\alpha /$ NF- $\kappa$ B/STAT3/RANTES signaling pathway described in mice is conserved in humans during angioplasty and stenting procedures. However, the advent of highthroughput genomics and chemical screening technologies will undoubtedly stimulate the pace at which such discoveries are put into practice.

\section{Acknowledgments}

This work is supported by NIH grants HL67330 and HL70694 (to T. Hla) and a postdoctoral fellowship from the American Heart Association (to M.L. Oo).

Address correspondence to: Timothy Hla, Center for Vascular Biology, Department of Pathology and Laboratory Medicine, Weill Medical College of Cornell University, 1300 York Avenue, Box 69, Room A607E, New York, New York 10065, USA. Phone: (212) 746-9953; Fax: (212) 746-2830; E-mail: tih2002@med.cornell.edu. 
1. Libby P, Schwartz D, Brogi E, Tanaka H, Clinton SK. A cascade model for restenosis. A special case of atherosclerosis progression. Circulation. 1992;86(suppl 6):III47-III52.

2. Schwartz SM. Smooth muscle migration in atherosclerosis and restenosis. JClin Invest. 1997;100(suppl 11):S87-S89.

3. Ross R. Atherosclerosis - an inflammatory disease. NEngl J Med. 1999;340(2):115-126.

4. Hansson GK. Atherosclerosis - an immune disease: The Anitschkov Lecture 2007. Atherosclerosis. 2009;202(1):2-10.

5. Groschel K, Schnaudigel S, Pilgram SM, Wasser K, Kastrup A. A systematic review on outcome after stenting for intracranial atherosclerosis. Stroke. 2009;40(5):e340-e347.
6. Charo IF, Ransohoff RM. The many roles of chemokines and chemokine receptors in inflammation. N Engl J Med. 2006;354(6):610-621.

7. Mallat Z, Taleb S, Ait-Oufella $\mathrm{H}$, and Tedgui A. The role of adaptive $\mathrm{T}$ cell immunity in atherosclerosis. J Lipid Res. 2009;50(suppl):S364-S369.

8. Tedgui A, Mallat Z. Anti-inflammatory mechanisms in the vascular wall. Circ Res. 2001;88(9):877-887.

9. Kovacic JC, et al. Stat3-dependent acute Rantes production in vascular smooth muscle cells modulates inflammation following arterial injury in mice. J Clin Invest. 2010;120(1):303-314.

10. Nabel EG, et al. Cell cycle signaling and cardiovascular disease. Cold Spring Harb Symp Quant Biol. 2002;67:163-170.

11. Kumar MS, Owens GK. Combinatorial control of smooth muscle-specific gene expression. Arterioscler Thromb Vasc Biol. 2003;23(5):737-747.

12. Ghosh S, Hayden MS. New regulators of NFkappaB in inflammation. Nat Rev Immunol. 2008;8(11):837-848.

13. Auffray C, et al. Monitoring of blood vessels and tissues by a population of monocytes with patrolling behavior. Science. 2007;317(5838):666-670.

14. Nachman RL, Rafii S. Platelets, petechiae, and preservation of the vascular wall. $N$ Engl J Med. 2008;359(12):1261-1270.

15. Cyster JG. Specifying the patterns of immune cell migration. Novartis Found Symp. 2007;281:54-61; discussion 61-54, 208-209.

16. Rastogi A, Stavchansky S. Drug eluting stents and beyond. Curr Pharm Des. 2008;14(21):2111-2120. 